\title{
CORRESPONDENCE
}

\section{THE USE OF A CYLINDER IN THE SLIT-LAMP OPTICAL SYSTEM}

\section{To the Editor of The British Journal of Ophthalmology}

SIR,-I see that Dr. Koby, in his book on " Slit-Lamp Microscopy of the Living Eye," refers on page 13 to "The Astigmatic Beam of the Author," quoting an article by himself on the subject in Rev. gén d'Ophtal., 1925, and the necessary appliance as having been made for him by the Optical and Mechanical Works, Basle. It is a matter of no importance, but the coincidence of this independent suggestion of Dr. Koby's is, incidentally, not the first. Towards the end of 1923, I experimented with the use of a plano-convex cylinder for creating an astigmatic light-bundle, and Messrs. Atha \& Co., in London, were kindly of assistance to me in the matter. At the beginning of 1924, I communicated the design for such a cylinder, together with plans of other devices, to Dr. O. Henker, Director of Messrs. Zeiss's Med. Optical Dept. In the summer of 1924, Dr. Henker showed me and furnished the model which it was proposed should be supplied in accordance with my specification. My cylinder is mounted on a hinged support by which it is attached to, so that it can always remain on, the slit-lamp arm; once the axis has been adjusted-and this must be done critically - the cylinder can be turned in or out of position with ease according as its use may be desired or not. If I may say so the fitting of such a cylinder into the end of the Koeppe tube, as Dr. Koby has done, is not very suitable for various reasons, one being that every time the cylinder is replaced for use the careful checking of the axis-adjustment must be repeated. A special independent simple diaphragm for attaching to the slit-lamp arm, abolishing the necessity to possess the Koeppe diaphragm, is made for use with or without my cylinder attachment.

In November, 1924, I sent the following typed instructions to Messrs. Zeiss to issue for use with my cylinder-

"(1) Adjust the slit-lamp in the usual way, so that the image of the lamp filament is in sharp focus on the diaphragm of the focussing lens.

(2) Put the cylinder-attachment on the slit-lamp arm a little in front of the mid-point between the slit and the focussing-lens, with the plain surface facing the slit. Turn the cylinder down (i.e. horizontal) out of the path of the rays whenever it is desired to adjust the focussing of the filament-image on the focussing-lens.

(3) With the cylinder in position, make the slit very narrow and examine, through the microscope (Oc. 2, Obj. A2) the narrow corneal stripe, or the anterior lens-capsule stripe. If these cannot 
be formed in sharp focus, rotate the cylinder in its mount, whilst still looking through the microscope, until the slit-images, focussed in the eye are seen sharply defined.

(4) Use the attachment with the 7.0 or 10.0 focussing-lens for the cornea and anterior chamber. With the $7.0 \mathrm{~cm}$. lens the length of the slit-image stripe falls a little short of the full diameter of the cornea. For the examination of the cornea and anterior chamber it is advantageous to use the F55 objectives in conjunction with the cylinder-attachment in order to detect abnormal curvatures of the cornea and abnormalities of the chamber-depth-local or general. The image-stripe on the iris and lens forms thus an arc to the curved image-stripe on the cornea, like the string to a bow.

(5) For the separation-lines of the lens-zones the cylinderattachment gives the best effect with the $7.0 \mathrm{~cm}$. focussing lens. A comprehensive view which, when the pupil is dilated, includes the periphery of the lens-zones is obtained by its use. Critical focussing is of course necessary, especially when the $7.0 \mathrm{~cm}$. lens is used."

In December, 1924, Messrs. Zeiss despatched to the United States six such cylinders, with special diaphragms, for my use in the Department of Ophthalmology, Graduate School of Medicine, University of Pennsylvania.

Yours truly,

BASIL GRAves.

LONDON.

\section{NOTES}

THE following appointments have been made

Appointments to St. Paul's Eye Hospital, Liverpool :Honorary Surgeons: P. Eldon Gorst, F. C. Plummer, Basil Graves; Honorary Assistant Surgeons: H. R. Bickerton, E. Nicholas Hughes, I. H. Moorhouse.

Mr. F. G. Thomas has been appointed additional Medical Referee under the Workmen's Compensation Act to take ophthalmic cases arising in the County Courts in Circuits Nos. 24, 30, and 31.

Mr. D. V. GIRI has been appointed Hon. Surgeon to the Royal Eye Hospital, Eastbourne.

IT has been decided for the future that the

Index annual index shall be issued with the December number. This will involve the postponement of the day of issue to subscribers from December 1 to December 7 or thereabouts. 